\title{
Energy efficiency digital filter design for spectral analysis of primary electrical values
}

\author{
Vladimir Mladenovic ${ }^{1}$, Caslav Stefanovic ${ }^{2}$, and Sergey Makov ${ }^{3, *}$ \\ ${ }^{1}$ Faculty of Technical Sciences in Cacak, University of Kragujevac, Sv. Save 65, Cacak, Serbia \\ ${ }^{2}$ Faculty of Natural Sciences and Mathematics, University of Pristina, K. Mitrovica, Serbia \\ ${ }^{3}$ Don State Technical University, Shevchenko 147, Shakhty 346500, Rostov region, Russia
}

\begin{abstract}
In this paper, the knowledge based design of digital filter for analysis of spectral components is illustrated. The primary electrical values are analyzed observing of the faults which appear by earth short circuits. The main point of view is negative sequence component and higher harmonics in distributed electrical networks. Method of symbolic processing is used to design digital filter that realizes negative sequence component. Designing of digital filter is based on active analog filter in the three-phase electrical networks using bilinear transformation. We use methods of computer algebra tools (Mathematica) to simulate processes in network. Also, we automatically derive properties of digital filter, and the knowledge embedded in symbolic expressions was used to simulate an example system. The characteristics of digital filter are given and annotated using different sampling frequency and entire cases of earth short circuits.
\end{abstract}

\section{Introduction}

Very often, the electrical networks are observed as four access ports in regard to analysis in some moment. Distribution of power electricity is realized using by three-phase system and analysis gets different form in power systems. In the ideal case, the power electricity is supplied on frequency by $50 \mathrm{~Hz}$ (or $60 \mathrm{~Hz}$ ) depending on the country where is distributed [1]. Also, on mentioned frequency the final voltage of $220 \mathrm{~V}$ (or $110 \mathrm{~V}$ ) is delivered to the customer/user. Because of the many different types of consumers, power systems possess different distortions or disturbances which are reflected in the form of higher harmonics. In this paper closer explanations about these distortions are given, how they are partially eliminated and how they affect the overall electricity supply [2]. Also, the most often faults that occur in electricity networks are short circuits as consequence of human factors, and often as consequence of outdated insulation. In this paper, earth short circuits are analysed [3].

In this paper, a new contemporary active approach as a solution for detecting earth short circuits using the third and fifth harmonics in three-phase AC network is proposed. For this method the digital filter is designed using algorithms by negative sequence component. The

\footnotetext{
${ }^{*}$ Corresponding author: makovserg@yandex.ru
} 
most often case of analysis is that electrical networks is as four poles. One of the methods for analyzing electrical networks is using contemporary digital signal processing [4].

The paper is organized as follows. Sections $2-5$ describe the general statement concept of three-phase system, symbolic processing tools, digital filters and interactive drawing of model system. Section 6 and 7 describe and analysis of system and system analysis. Sections 8 and 9 devote an implementation digital filter for obtaining negative sequence component and show results of the system analysis and modeling using schematic representation of systems, as well as cases of the earth short circuits.

\section{Form three phases}

Most power systems consist of three phases. A few reasons deserve attention for threephase phenomena [5], [6]: (a) the system voltages are almost balanced in normal operation; (b) the most of devices are single phase which use; (c) three-phase models increase the complexity of the approach in some cases significantly.

The most disturbances are not balanced as they are deviations from the ideal case. On the other side, modern adjustable devices described in [7] generate variable frequencies that are transmitted through power system and influence on the power supply with dominant nonlinear characteristics. These frequencies generate higher harmonics as a consequence of variability. Harmonics are produced in the process and can be modeled as harmonic current sources [8]. There are two mechanisms through higher harmonic are generated. The first mechanism is the rectifier operation, which injects harmonic currents into the supply system by an electronic switching process. The second mechanism is the inverter operation [9]. Higher harmonics cause heating effects in machines, both in the conductors and in the iron. In particular, eddy current losses are proportional to the square of the frequency. Further, some harmonics are negative phase sequence and these give rise to additional losses by inducing higher frequency currents in machine rotors [10].

In this paper, the third and fifth harmonics are significant for analysis. The third harmonic does not propagate through the distribution transformer. The presence of a high third-harmonic voltage is in almost all cases due to load supplied from the local distribution transformer. The fifth harmonic spreads through transformers and is thus related to the amount of harmonic load over a much wider area. The measured television peak for the third and fifth harmonics occurred for most days at about the same time. However, the third-harmonic distortion started to drop up to $1 \mathrm{~h}$ before the fifth harmonic for some days , pointing to a difference in local viewing patterns compared to the average over a wider area [11], [12].

\section{Signal processing tools}

In signal processing the time-frequency domain has often been exploited for analyzing signals with fast changing spectral contents [13], [14]. Many of algorithms are used in analysis of power quality monitoring [15], [16]: Root Mean Square value, (Voltage Magnitude Variations, Voltage Frequency Variations, Three-Phase Unbalance), Fourier Analysis (Short Time Fourier Transform analysis), Wavelet Transform, S-Transform, Park's Vector Approach, Kalman filters, Detection of faults [17], [18]. 


\section{Symbolic processing and digital filter design using Mathematica}

Modern research in the field of engineering science often includes two phases measurements and simulations. With comparable results from both phases relevant conclusions can be drawn for the implementation and/or further investigation. Very often, the numerous input numeric parameters, as set in the simulation process, lead to disagreement in the results [19]. Some of the reasons for these disagreements can be a finite data word-length and the imperfections of certain numerical tools and algorithms [20]. The experience often shows an inconsistency of measured and simulated results that can be very confusing for researchers. One of the ways to overcome accuracy problems in simulations and processing is to use symbols instead of numbers, so that the influence of symbolic parameters can be easily recognized in the final result. Symbolic processing may play an important role in education for better understanding of the nature of the analyzed phenomena. Symbolic processing is a unique feature that performs computations as closedform expressions in terms of system parameters kept as symbols [21].

The Mathematica is a general computer software system and language intended for mathematical applications. It can be used as a numerical and symbolic calculator, a visualization system, a high-level programming language, modeling and data analysis environment, a system for representing knowledge, a software platform and a way to create interactive documents that mix text, graphics, sound and formulas.

\section{Digital filters}

Many examples testify about very wide applications of digital filters. The following list gives some of the main advantages of digital over analog filters. A digital filter is programmable. An analog filter can only be changed by redesigning the filter circuit. Digital filters are easily designed, tested and implemented on a general-purpose computer or workstation [22]. Digital filters are very much more versatile in their ability to process signals in a variety of ways; this includes the ability of some types of digital filter to adapt to changes in the characteristics of the signal. Fast DSP processors can handle complex combinations of filters in parallel or cascade (series), making the hardware requirements relatively simple and compact in comparison with the equivalent analog circuit.

The SchematicSolver, as part of Mathematica, provides an easy point-and-click interface for performing the most common drawing tasks. The SchematicSolver describes a system as a symbolic object in the form of a list of elements [23], [24].

\section{Analysis of system}

Analyzing all cases of earth short circuits, it notices that six types of earth short circuits exist. According to them the zero component can be described in form:

$$
s_{m}(t)=\operatorname{Re}\left\{u(t) \cdot \exp \left[j\left(\omega_{c} t+\frac{2 \pi}{M}(m-1)+\lambda\right)\right]\right\}, m=1,2, \ldots, M
$$

where $\lambda$ - initial phase and $M$ - the number of phases in constellation, $\omega_{c}$ - network frequency and $u(t)$ - form of voltage of three-phase system. 
According to number cases of earth short circuit it is easy to define initial phase and the number of phases, $\lambda=\pi / 3$ and $M=6$. The case when the earth short circuit arises simultaneously on all phases is not analyzed. In this case the system will activate all circuit breakers.

\section{Description and system analysis}

The character of present components in electrical network can be described using vector operator $\dot{a}$ (versor $\dot{a}$ ). Versor $\dot{a}$ is a complex vector when the absolute value equals to one and shows determinated corner position. Using Euler's expressions a few characteristical versors can be shown $\varphi=120^{\circ}$ and $\varphi=240^{\circ}$, so $\varphi=120^{\circ} \equiv 2 \pi / 3$, that is $e^{j \frac{2 \pi}{3}}=\cos \frac{2 \pi}{3}+j \sin \frac{2 \pi}{3}=-\frac{1}{2}+j \frac{\sqrt{3}}{2}=\dot{a}, \quad \varphi=240^{\circ} \equiv 4 \pi / 3=2(2 \pi / 3), \quad$ that $\quad$ is $e^{j \frac{4 \pi}{3}}=\cos \frac{4 \pi}{3}+j \sin \frac{4 \pi}{3}=-\frac{1}{2}-j \frac{\sqrt{3}}{2}=\dot{a} \cdot \dot{a}=(\dot{a})^{2}$ and there are important for further analysis.

Using further analysis we obtain $\dot{a} \cdot \dot{a} \cdot \dot{a}=(\dot{a})^{3}=e^{j 2 \pi}=1$. From the last expression conclusion can be derivated, that is $1+\dot{a}+(\dot{a})^{2}=0$. Further, we will use the label " $a$ " without a upper point for ease of writing. In every power system the different unsymmetries are present because the different electromagnetic values are present. These nonsymmetries can cause changing of phases, amplitudes of voltage or current and influence the process of power distribution energy. Very often, unsymmetrical system is adapted to become symmetrical system in practice and it can be used in further different calculations. So, the signals create symmetrical system thus obtaining symmetrical components:

Direct order

$$
U_{d}=\frac{1}{3}\left(U_{A}+a \cdot U_{B}+a^{2} \cdot U_{C}\right)
$$

Negative sequence order

$$
U_{i}=\frac{1}{3}\left(U_{A}+a^{2} \cdot U_{B}+a \cdot U_{C}\right)
$$

Zero order

$$
U_{o}=\frac{1}{3}\left(U_{A}+U_{B}+U_{C}\right)
$$

where $U_{A}, U_{B}$ and $U_{C}$ show the signals shifted in vector diagram of three-phase system.

The phases are $2 \pi / 3$ and modules are equal each other in a symmetrical system.

Digital filter is designed according to analog filter which is obtained by algorithm of negative sequence component. All combinations of earth short circuits are caused when the third and fifth harmonics are present in the networks And according to them we detect which kind of fault occurs. 


\section{Description of filter for obtaining negative sequence component}

The figure 1 shows $\mathrm{RC}$ active continual analog filter for obtaining negative sequence component. In this case, the high pass filter is used as basic of this analog filter.

The analog and digital filters posses a few joint features: (a) they have equal number of poles; (b) digital filter is stable if the analog filter is stable; (c) the frequency characteristic of digital filter is periodic; (d) frequency characteristic of analog filter; (e) impulse response of cascade of analog filters is not the same as the impulse response of cascade of digital filters.

The bilinear transformation is used for copying from analog to digital domain because it has very good features.

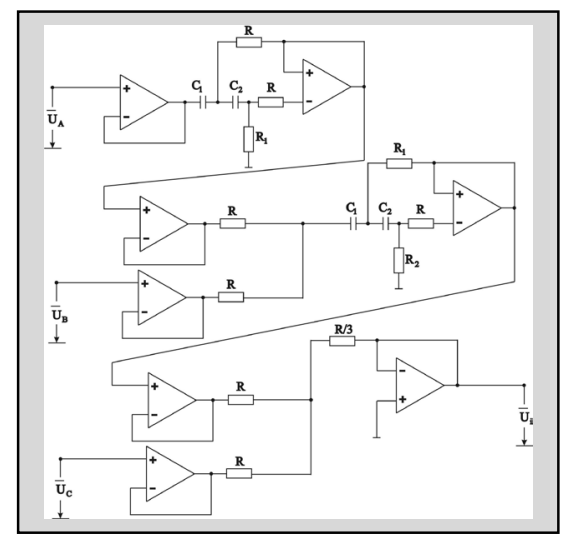

Fig. 1. Prototype of active analog filter for obtaining negative sequence component

The transfer function of one section of filter is described in the frequency domain:

$$
H_{1}(s)=\frac{s \cdot C_{1} C_{2} R_{1} R_{2}}{1+s \cdot R_{1}\left(C_{1}+C_{2}\right)+s^{2} \cdot C_{1} C_{2} R_{1} R_{2}}
$$

\subsection{Transformation to digital system}

The voltages $\overline{U_{A}}, \overline{U_{B}}$ and $\overline{U_{C}}$ are input voltages which are phase shifted $2 \pi / 3$. The phase between every voltage is equal and conclusion is that the system is symmetrical. Nominal frequency is $\mathrm{f}=50 \mathrm{~Hz}$ in network. If $R_{1}=R_{2}=R$ and $C_{1}=C_{2}=C$ then

$$
\frac{1-\omega^{2} \tau^{2}}{\omega \tau^{2}}=\sqrt{3}
$$

so $\tau=R C=3.16 \cdot 10^{-3} s$, where is $\omega=2 \pi f$.

Using bilinear transformation (BT)

$$
s=\frac{2}{T_{S}} \cdot \frac{z-1}{z+1}
$$


where $T_{S}=\frac{1}{f_{S}}$, and $f_{S}$ is sampling frequency, the transfer function of one section of filter in z-domain can be obtained:

$$
\begin{gathered}
H_{1}(s) \stackrel{B T}{\longrightarrow}=H_{1}(z) \\
H_{1}(z)=a \cdot K\left(T_{S}\right) \cdot \frac{\left(1-2 \cdot z^{-1}+\cdot z^{-2}\right)}{1+\alpha\left(T_{S}\right) \cdot z^{-1}+\beta\left(T_{S}\right) \cdot z^{-2}}
\end{gathered}
$$

where $K\left(T_{S}\right)=\frac{1}{T_{S}^{2}+4 \cdot \tau \cdot T_{S}+4 \cdot \tau^{2}}, a=4 \tau^{2}, \alpha\left(T_{S}\right)=\frac{2 \cdot T_{S}^{2}-8 \cdot \tau^{2}}{T_{S}^{2}+4 \cdot \tau \cdot T_{S}+4 \cdot \tau^{2}}$ and $\beta\left(T_{S}\right)=\frac{T_{S}^{2}-4 \cdot \tau \cdot T_{S}+4 \cdot \tau^{2}}{T_{S}^{2}+4 \cdot \tau \cdot T_{S}+4 \cdot \tau^{2}}$.

Figure 2 shows section of digital filter which is obtained using bilinear transformation.

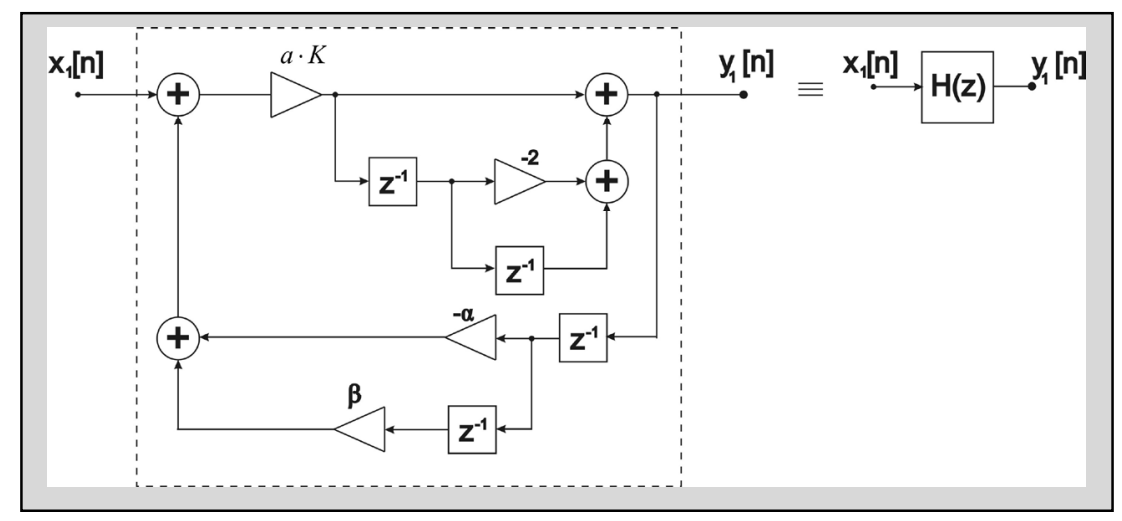

Fig. 2. Caption of the Figure 1. Below the figure.

It is very important to carry out transformation input analog values in z-domain for getting a total transfer function. That is:

$$
\begin{gathered}
\overline{U_{B}}=U \cos (\omega t) \stackrel{n}{\longrightarrow} x_{1}[n] \stackrel{z}{\longrightarrow} x_{1}(z) \\
\overline{U_{C}} \angle 2 \pi / 3 \stackrel{z}{\longrightarrow} H_{\varphi_{1}}(z)=x_{2}(z)=-\frac{1}{2} \cdot x_{1}(z)\left(1-\sqrt{3} \cdot z^{-1}\right) \\
\overline{U_{A}} \angle 4 \pi / 3 \stackrel{z}{\longrightarrow} H_{\varphi_{2}}(z)=x_{3}(z)=-\frac{1}{2} \cdot x_{1}(z)\left(1+\sqrt{3} \cdot z^{-1}\right)
\end{gathered}
$$

Using expressions (8)-(12) the total transfer function is in form:

$$
H_{e k v}(z)=\gamma \cdot H_{1}^{2}(z)-0.5 \cdot \gamma \cdot H_{1}(z)+1.73 \cdot \gamma \cdot H_{1}(z) \cdot z^{-1}-0.5 \cdot \gamma-1.73 \cdot \gamma \cdot z^{-1}
$$

On the basis of expression (13) figure 3 shows digital filter for obtaining negative sequence component. 


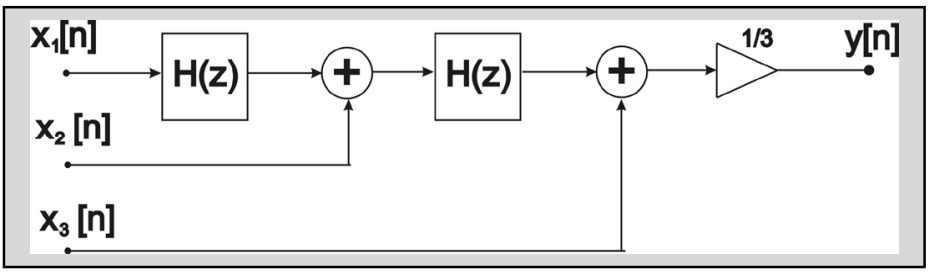

Fig. 3. Digital filter that realize negative sequence component

\section{Earth short circuits cases}

Table 1 shows all cases of ESC (Earth Short Circuits). Every component can be described using expression (1). We assume that the system works in normal mode and in this case the zero component is equal zero. But, when the noises are present disregarding the mode (normal or mode of earth short circuits) in the symmetrical system.

Table 1. The cases of ESC in three-phase electrical network

\begin{tabular}{|c|c|c|c|c|}
\hline $\boldsymbol{U}_{\boldsymbol{A}}$ & $\boldsymbol{U}_{\boldsymbol{B}}$ & $\boldsymbol{U}_{\boldsymbol{C}}$ & $\boldsymbol{\varphi}_{\boldsymbol{0}}$ & $\boldsymbol{U}_{\boldsymbol{0}}$ \\
\hline 0 & 0 & $E S C$ & $\pi / 3$ & $\frac{1}{2}+j \frac{\sqrt{3}}{2}$ \\
\hline 0 & $E S C$ & 0 & $5 \pi / 3$ & $\frac{1}{2}-j \frac{\sqrt{3}}{2}$ \\
\hline 0 & $E S C$ & $E S C$ & 0 & 1 \\
\hline$E S C$ & 0 & 0 & $\pi$ & -1 \\
\hline$E S C$ & 0 & $E S C$ & $2 \pi / 3$ & $-\frac{1}{2}+j \frac{\sqrt{3}}{2}$ \\
\hline$E S C$ & $E S C$ & 0 & $4 \pi / 3$ & $-\frac{1}{2}-j \frac{\sqrt{3}}{2}$ \\
\hline
\end{tabular}

Figures 4, 5, 6, 7, 8, 9, 10, 11 and 12 show spectral components on the output of the system according to earth short circuits on phases $A, B, C, A-B, A-C$ and $B-C$.

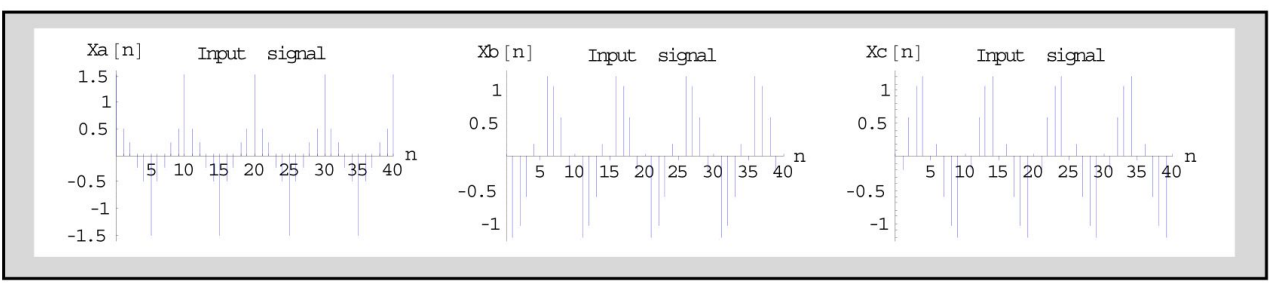

Fig. 4. Forms of input signal in digital filters system 


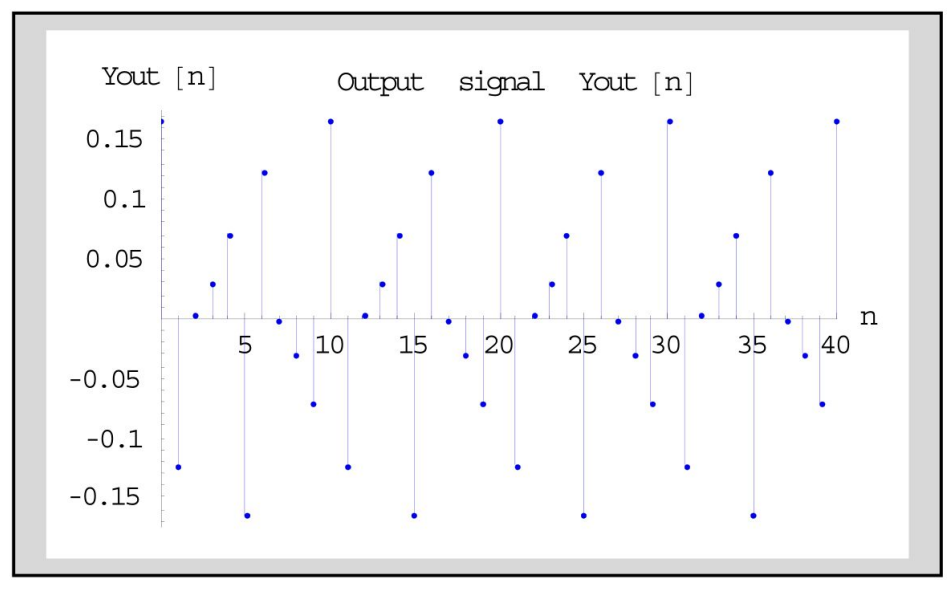

Fig. 5. Response of output signal on the output of digital filters system.

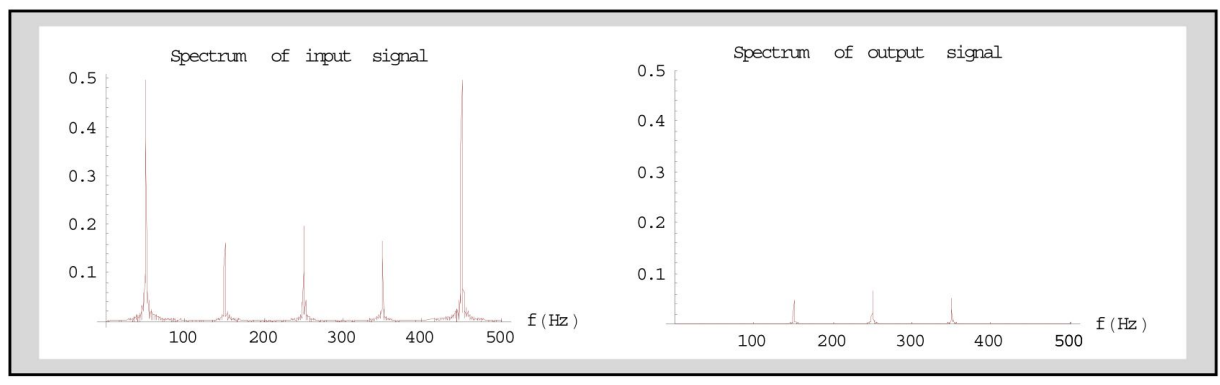

Fig. 6. Spectrum of input (left side) and output signal (right side) in the normal mode without faults

The models of ideal earth short circuits are used in analysis, modelled with coefficients $K_{1}, K_{2}$ and $K_{3}$ which have value zero in case of earth short circuit, and value 1 where the earth short circuit does not exist. Numerical values of spectral components and phases for first, third and fifth harmonic for all cases are printed in table 2.

Table 3. Values of spectral components and phases when the third and fifth harmonics are present with frequency sampling of $f_{S}=500 \mathrm{~Hz}$.

\begin{tabular}{|c|c|c|c|c|c|c|c|c|}
\hline \multicolumn{2}{|c|}{ Cases in network } & \multicolumn{6}{|c|}{ Frequency sampling in system $f_{s}=\mathbf{5 0 0 H z}$} \\
\cline { 4 - 9 } & $\begin{array}{c}\text { Basic harmonic } \\
f_{0}=\mathbf{5 0 H z}\end{array}$ & \multicolumn{2}{|c|}{$\begin{array}{c}\text { Third harmonic } \\
f_{3}=\mathbf{1 5 0 H z}\end{array}$} & \multicolumn{2}{c|}{$\begin{array}{c}\text { Fifth harmonic } \\
f_{5}=\mathbf{2 5 0 H z}\end{array}$} \\
\hline$K_{3}$ & $K_{2}$ & $K_{1}$ & $A_{0}$ & $\varphi_{0}$ & $A_{3}$ & $\varphi_{3}$ & $A_{5}$ & $\varphi_{5}$ \\
\hline 1 & 1 & 1 & 0 & - & 25,7514 & $15,5225^{\circ}$ & 33,3333 & $0^{\mathbf{o}}$ \\
\hline 1 & 1 & 0 & 83,3333 & $60^{\circ}$ & 7,50252 & $113,284^{\circ}$ & 0 & $180^{\mathbf{o}}$ \\
\hline 1 & 0 & 1 & 83,3333 & $180^{\circ}$ & 53,5292 & $15,5225^{\circ}$ & 66,6667 & $0^{\circ}$ \\
\hline 0 & 1 & 1 & 83,3333 & $-60^{\circ}$ & 7,50252 & $-82,2388^{\circ}$ & 0 & $180^{\circ}$ \\
\hline 1 & 0 & 0 & 83,3333 & $120^{\circ}$ & 27,7778 & $31,045^{\circ}$ & 33,3333 & $0^{\circ}$ \\
\hline 0 & 1 & 0 & 83,3333 & $0^{\circ}$ & 27,7778 & $-164,478^{\circ}$ & 33,3333 & $180^{\circ}$ \\
\hline 0 & 0 & 1 & 83,3333 & $-120^{\circ}$ & 27,7778 & $0^{\circ}$ & 33,3333 & $0^{\circ}$ \\
\hline
\end{tabular}




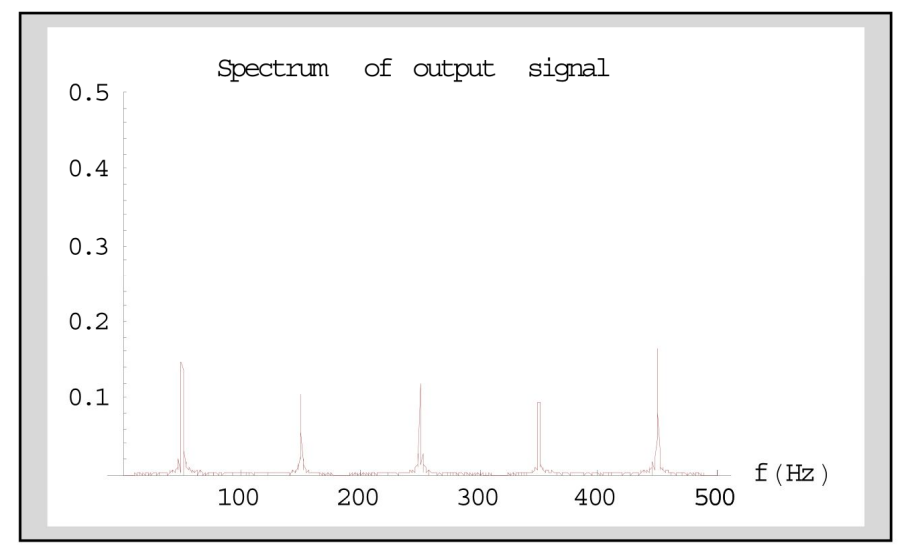

Fig. 7. Response and spectrum of output signal with fault $\mathrm{A} \Rightarrow K_{1}=0 ; K_{2}=1 ; K_{3}=1$

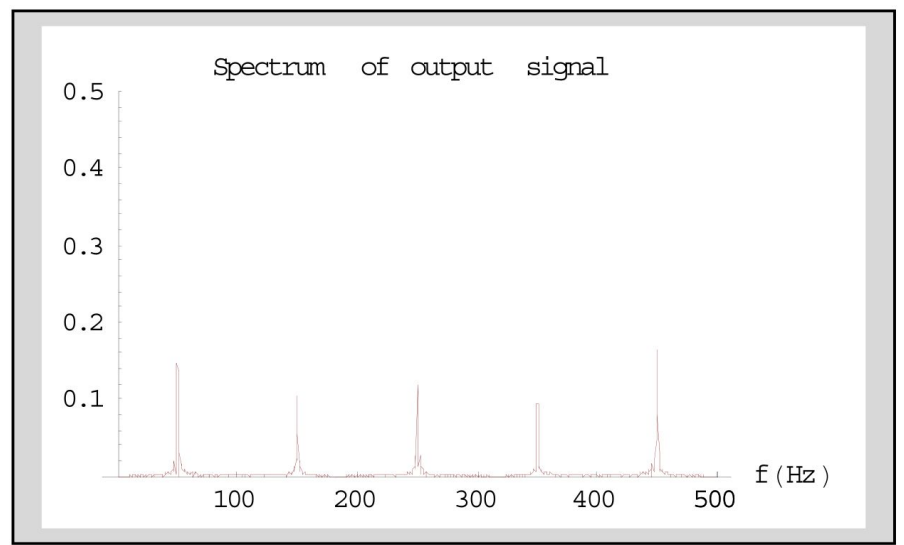

Fig. 8. Response and spectrum of output signal with fault $\mathrm{B} \Rightarrow K_{1}=1 ; K_{2}=0 ; K_{3}=1$

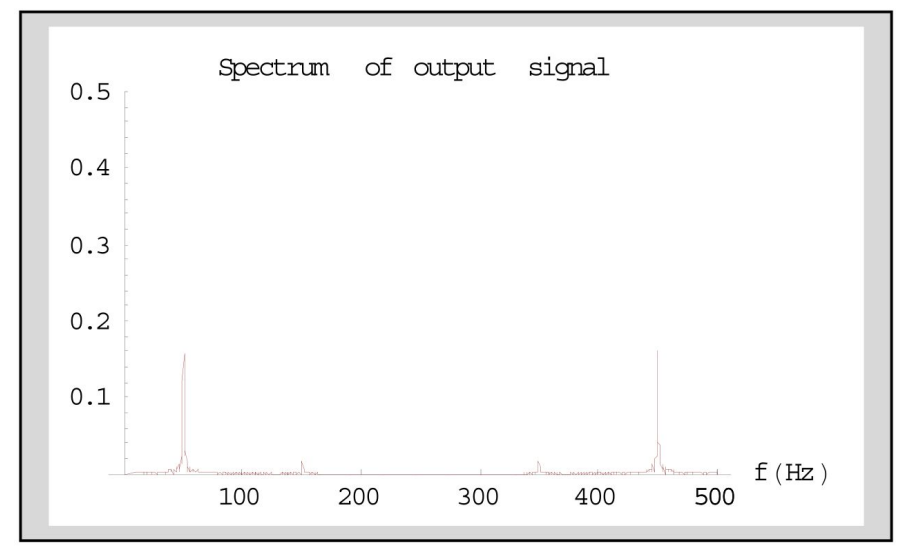

Fig. 9. Response and spectrum of output signal with fault $\mathrm{C} \Rightarrow K_{1}=1 ; K_{2}=1 ; K_{3}=0$ 


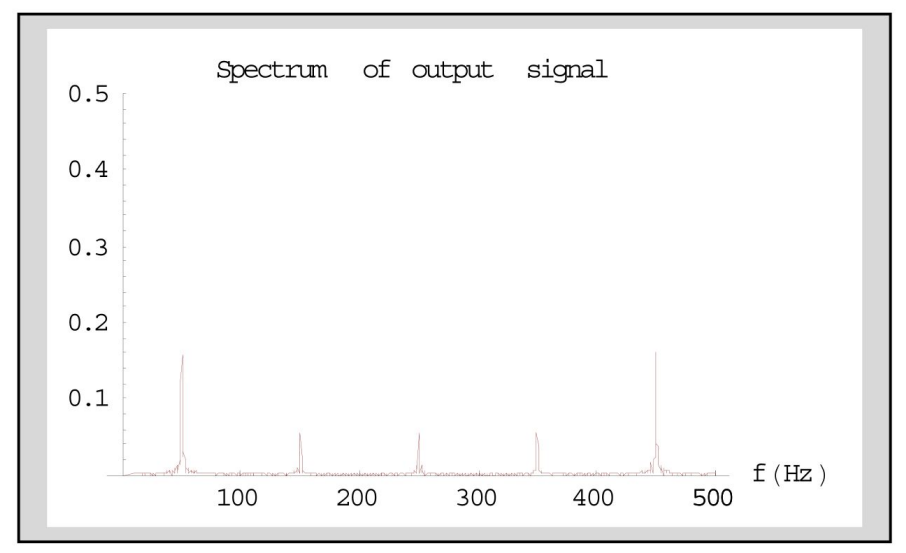

Fig. 10. Response and spectrum of output signal with fault A-B $\Rightarrow K_{1}=0 ; K_{2}=0 ; K_{3}=1$

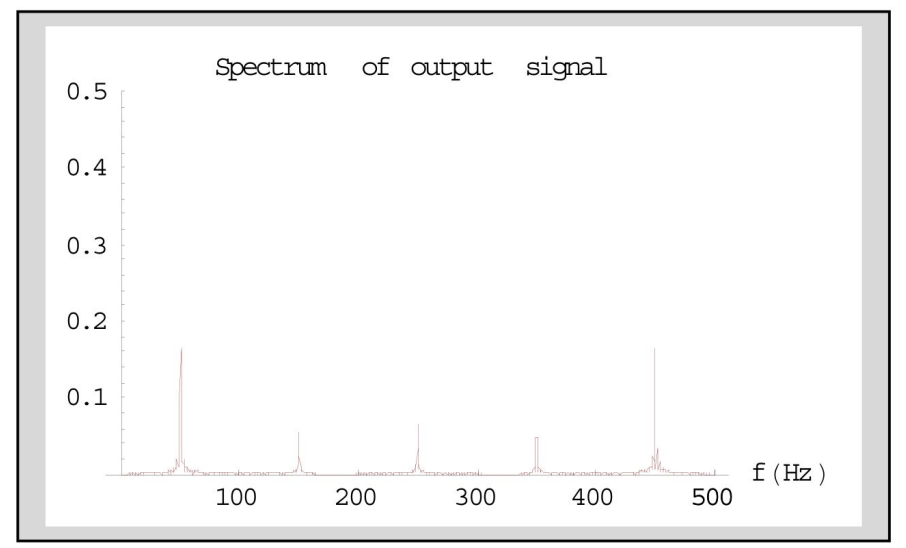

Fig. 11. Response and spectrum of output signal with fault $\mathrm{A}-\mathrm{C} \Rightarrow K_{1}=0 ; K_{2}=1 ; K_{3}=0$

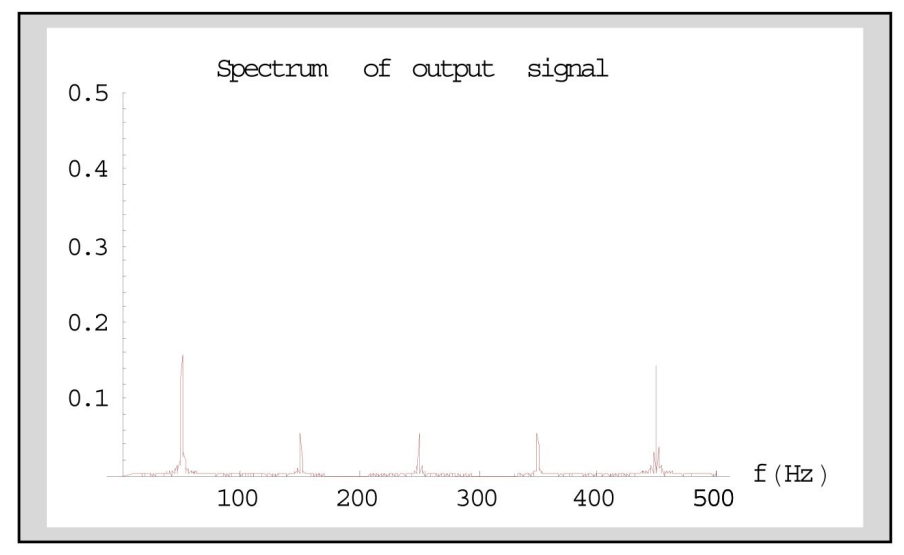

Fig. 12 Response and spectrum of output signal with fault B-C $\Rightarrow K_{1}=1 ; K_{2}=0 ; K_{3}=0$ 


\section{Conclusion}

In this paper, a new approach for detecting earth short circuits is presented. To detect earth short circuits, presence of the third and fifth harmonics is used. For this needs the digital filter is designed on the basis of the negative sequence component which is present in all the three-phase systems. The third and fifth harmonic can show in detail the presence of a fault in the electrical network. Since the moments of earth short circuits are finite, using digital signal processing methods can perform many activities necessary to remedy the faults. Also, all the processes and design are performed using by symbolic processing tools. This method can be applied in other areas of process control data acquisition and processing, such as gas, oil and other industries.

This work is supported in part by the Ministry of Science of Serbia under Grant TR32023.

\section{References}

1. T. Short, Electric power distribution handbook (CRC Press LLC, 2004)

2. M. Bollen, I. Gu, Signal processing of power quality disturbances, IEEE Press (2006)

3. A. Pansini, Electrical distribution engineering, 3rd Edition (CRC Press, Piscataway, 2007)

4. D. Patrick, S. Fardo, Electrical distribution systems, 2nd Edition (CRC Press, 2009)

5. N. Mohan, T. Undeland, W. Robbins, Power Electronics (John Wiley, Chichester, 1995)

6. M. Bollen, Understanding Power Quality Problems-Voltage Sags and Interruptions (IEEE Press, Piscataway, 2000)

7. W. Xu, H. Dommel, M. Hughes, G. Chang, L. Tan, IEEE Trans. Power Delivery, 14 (2), 595 (1999)

8. R. Dugan, M. McGranaghan, H. Beaty, Electrical power systems quality (McGrawHill, New York, 1996)

9. G. Porter, J. Van Sciver, Power quality solutions: case studies for trouble-shooters (Fairmont Press, Lilburn, 1998)

10. G. Wakilen, Power Systems harmonics: fundamentals, analysis and filter design, Springer (2001)

11. J. Schonek, Technique Schneider Electric, 202, 2 (2001)

12. C. Collombet, J.-M Lupin, J. Schonek, ECT 152 first issue, Cahier Technique Schneider Electric, 1, 152, 2 (1999)

13. A. Gargoom, N. Ertugrul, W. Soong, European Conference on Power Electronics and Applications, IEEE, 10 (2005)

14. T. Vega, V. Riog, H. Segundo, 9th International Conference Electrical Power, IEEE (2007)

15. F. Jurado, N. Acero, B. Ogayar, Canadian Conference on Electrical and Computer Engineering, IEEE CCECE 2002, 1, 82 (2002)

16. U. N. Khan, In Proc. 8th Int. Conf. Environ. Electr. Eng. (EEEIC), 1 (2009)

17. O. Chaari, M. Meunier, F. Brouaye, IEEE Trans. Power Delivery, 11, 3, 1301 (1996)

18. W. Zhao, Y. Song, Y. Min, Electric Power Systems Research, 53, 1, 23 (2000) 
19. V. Mladenovic, D. Porrat, M. Lutovac, 10th Int. Conf. on Telecommunications in Modern Satellite, Cable and Broadcasting Services, 1, 265 (2011)

20. M. Lutovac, D. Tošić, International Journal of Control, Special Issue on Symbolic Computing in Control, 79, 11 (2006)

21. M. Trott, The mathematica guidebook for symbolics (Springer, Illinois, 2006)

22. M. Lutovac, J. Certic, Lj. Milic, Circuits Syst Signal Process, 29, 1, 51 (2010) DOI 10.1007/s00034-009-9119-2

23. M. Lutovac, D. Tošić, SchematicSolver Version 2.2, (2010) [Online] Available: $\mathrm{http}$ ///books.google.com/books?id=9ue-uVG_JsC

24. D. Tošić, M. Lutovac, The IPSI BgD Transactions on Advanced Research, 3, 1 (2007) 